\title{
INFLUÊNCIA DA POPULAÇÃO FLUTUANTE NO PLANEJAMENTO DO SISTEMA DE LIMPEZA URBANA
}

Paulo Tárcyo Resende Teixeira ${ }^{1}$, Jose Antonio Rodrigues Souza ${ }^{2}$, Débora Astoni Moreira ${ }^{3}$, Aline Maria Souza Viana $^{4}$, Joceline Maria Costa Soares ${ }^{5}$

\section{RESUMO}

Uma gestão adequada se inicia com a determinação das características dos resíduos gerados “in loco" para que se possa realizar um planejamento eficaz, uma vez que dados de literatura podem não corresponder à realidade local. Assim, neste trabalho, objetivou-se determinar as características quantitativas e qualitativas dos resíduos sólidos produzidos no município de Urutaí-GO, bem como estudar o efeito da sazonalidade populacional e valoração desses resíduos. De acordo com os resultados, verificou-se que os resíduos sólidos, durante o período letivo do IFGoiano Campus Urutaí, são compostos por 10,47\% de plásticos; 4,29\% de metais; 4,13\% de vidros; $13,17 \%$ de papéis e $61,11 \%$ de material de descarte, ocorrendo reduções de 34,99\%; 55,35\%; 39,39\% 36,72\% nas quantidades de plásticos, metais, vidros e de papéis, respectivamente, bem como incremento de $18,17 \%$ na quantidade de material de descarte durante o período de férias escolares. A geração per capta, peso específico aparente e o volume gerado mensalmente, reduziram

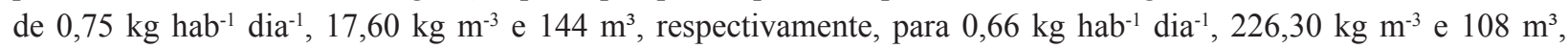
respectivamente, durante o período de férias escolares. Verificou-se, também, que a coleta seletiva é uma alternativa viável e autossustentável, sendo que sua ausência provoca um desperdício de 3,4 salários mínimos durante o período letivo e de 1,6 salários mínimos durante o período de férias escolares.

Palavras-Chave: Reciclagem, gerenciamento de resíduo sólido, gestão ambiental.

\section{ABSTRACT \\ INFLUENCE OF FLOATING POPULATION IN THE PLANNING OF THE URBAN CLEANING SYSTEM}

Adequate management begins with determining the characteristics of the waste generated "in loco" in order to carry out effective planning, since literature data may not correspond to local reality. Thus, this work aimed to determine the quantitative and qualitative characteristics of solid waste produced in the city Urutaí-GO, as well to study the effect of population seasonality and waste valuation. According to the results, during the IFGoiano Appropriate management begins with the determination of the characteristics of the generated waste "in loco" so that an effective planning can be carried out, since literature data may not correspond to the local reality. In this work, the objective was to determine the quantitative and qualitative characteristics of the solid waste produced in the city of Urutaí-GO, as well as to study the effect of population seasonality and the evaluation of these residues. According to the results, it was verified that solid waste, during the Campus Urutaí IFGoiano school year, consists of $10.47 \%$ of plastics; $4.29 \%$ of metals; $4.13 \%$ of glasses; $13.17 \%$ papers and $61.11 \%$ discard material, with reductions of $34.99 \% ; 55.35 \% ; 39.39 \% 36.72 \%$ in the quantities of plastics, metals, glasses and paper, respectively, as well as an increase of $18.17 \%$ in the amount of discard material during the school holidays. The per capita generation, apparent specific weight and volume generated monthly reduced from $0.75 \mathrm{~kg} \mathrm{hab}^{-1}$ day $^{-1}, 17.60 \mathrm{~kg} \mathrm{~m}^{-3}$ and $144 \mathrm{~m}^{3}$, respectively, to $0.66 \mathrm{~kg} \mathrm{hab}^{-1}$ day $^{-1}, 226,30 \mathrm{~kg} \mathrm{~m}^{-3}$ and $108 \mathrm{~m}^{3}$, respectively, during the school holidays. It was also verified that the selective collection is a viable and self-sustaining alternative, being that its absence causes a waste of 3.4 minimum wages during the school year and of 1.6 minimum wages during the period of school vacations.

Keywords: Recycling, solid waste management, environmental management

\section{Recebido para publicação em 12/04/2016. Aprovado em 16/09/2016.}

1 - Engenheiro Agrícola, IFGoiano - Campus Urutaí, paulotarcyoresende@gmail.com

2 - Engenheiro Agrícola, Professor IFGoiano - Campus Urutaí, jose.antonio@ifgoiano.edu.br

3 - Química, Professora IFGoiano - Campus Urutaí, debora.astoni@ifgoiano.edu.br

4 - Gestora Ambiental, Mestranda PPG-CRENAC, IFGoiano - Campus Urutaí, alinemsviana@hotmail.com>

5 - Gestora Ambiental, Mestranda PPG-CRENAC, IFGoiano - Campus Urutaí, jocelinecostasoares@gmail.com 


\section{INTRODUÇÃO}

Um dos grandes problemas atuais enfrentados pela sociedade, tem sido a destinação final inadequada dos resíduos sólidos gerados, que vem se agravando pelo crescimento da população e o consequente incremento da geração per capita. De acordo com a CEMPRE (2016), 82\% dos municípios brasileiros não desenvolvem programas de coleta seletiva, o que torna a busca por soluções a esta problemática, um dos principais desafios à gestão pública, que deve encontrar alternativas de minimização de resíduos e eliminação ambientalmente compatível, conservando os solos, as águas e o ar.

Considerada um dos setores do saneamento básico, a gestão dos resíduos sólidos não tem recebido atenção necessária por parte do poder público. Com isso, compromete-se cada vez mais a já combalida saúde da população, bem como degradam-se os recursos naturais, especialmente o solo e os recursos hídricos. A interdependência dos conceitos de meio ambiente, saúde e saneamento é evidente, reforçando a necessidade de integração das ações desses setores em benefício da qualidade de vida da população brasileira (LUSSARI et al., 2013).

A inexistência de um modelo adequado de gestão dos resíduos sólidos urbanos para as prefeituras tem criado sérios problemas, os quais, muitas vezes, se traduzem na agressão ao ambiente, o que acarreta no aumento dos casos de enfermidades e na má qualidade de vida das pessoas (SCACABAROSSI et al., 2012). De acordo com OLIVEIRA et al. (2016), $80 \%$ de todas as doenças e mais de um terço dos óbitos dos países em desenvolvimento são causados pelo consumo de água contaminada e em virtude da falta de saneamento básico.

Diante dessa problemática, é evidente a necessidade de se promover uma gestão adequada, a fim de prevenir ou reduzir os possíveis efeitos negativos sobre o meio ambiente, bem como os riscos à saúde humana. Levando-se em consideração esta necessidade, as medidas devem ser adotadas de modo a evitar o descarte ou a eliminação descontrolada dos resíduos. Assim, neste trabalho, objetivou-se caracterizar quantitativamente e qualitativamente os resíduos sólidos gerados no município de Urutaí - GO, bem como estudar o efeito da sazonalidade populacional e valoração desses resíduos.

\section{MATERIAL E MÉTODOS}

O trabalho foi conduzido em um lixão localizado no município de Urutaí-GO, com coordenadas geográficas de $17^{\circ} 43^{\prime} \mathrm{S}, 48^{\circ} 19^{\prime} \mathrm{W}$ e altitude de $855 \mathrm{~m}$, durante o período maio a agosto de 2014 . Para caracterização dos resíduos gerados, amostras foram coletadas nos dias $07 / 05 ; 16 / 05 ; 15 / 06$; 16/07; 27/07 e 08/08 de 2014, determinando-se a geração per capita, composição gravimétrica, peso específico aparente, umidade, compressividade e valoração dos resíduos, conforme metodologias descritas por MONTEIRO et al. (2001).

Incialmente, foram separadas amostras com cerca de três metros cúbicos de volume, os quais foram coletados a partir do resíduo não compactado (lixo solto), provindo de tratores coletores municipais. Essas amostras foram distribuídas sobre lona plástica, em área plana, onde, após serem desacondicionados, foram misturadas com o auxílio de pás e enxadas até obtenção de lote único homogêneo. Posteriormente, foram submetidas a quarteamentos sucessivos até obtenção do volume de 0,75 metros cúbicos em cada quarto, de onde foram separados os materiais aptos para comercialização: papel, plástico, metais, vidros e o material de descarte, que é o rejeito das amostras.

A determinação do peso específico aparente foi obtido com o auxílio de uma balança, por meio da diferença média entre o peso determinado antes e após enchimento de cinco latões, com volumes de 100 litros, previamente aferidos, com resíduos anteriormente quarteados. A partir do peso específico aparente e, conhecendo-se o volume de cada carreta e a quantidade de carretas descarregadas, determinou-se a quantidade diária $(\mathrm{kg})$ de resíduos sólidos conduzida ao lixão.

A umidade foi determinada a partir de amostras retalhadas com auxílio de facões, sendo determinado seu peso antes e após secagem em estufa a $105{ }^{\circ} \mathrm{C}$ até obtenção de peso constante. No total, foram coletadas cinco amostras com peso aproximado de 200 gramas.

$\mathrm{Na}$ composição gravimétrica, foram 
determinados os percentuais dos componentes recicláveis, de acordo com os interesses da administração municipal (plásticos, metais, vidros, papeis e descarte), espalhando-se todo o material contido nos latões sobre lona plástica, em área plana, determinando-se a porcentagem de cada componente em relação ao peso total da amostra.

A geração per capita foi obtida medindo-se o volume de resíduos encaminhados ao lixão durante um dia inteiro de trabalho, multiplicado pelo peso específico aparente determinado anteriormente e, dividindo-se pela população atendida (correspondente $80 \%$ da população urbana).

A compressividade foi determinada por meio da compactação dos resíduos em latões com volumes previamente aferidos, os quais apresentavam 100 litros de capacidade e altura de $0,43 \mathrm{~m}$, os quais foram cheios até o nível superior. A compressão foi aplicada com auxílio de um compactador de solo, cujo peso equivalente era de $15 \mathrm{~kg}$.

Para analisar a influência da sazonalidade na geração de resíduos, foi determinada geração per capita, composição gravimétrica, peso específico aparente, umidade e compressividade, durante o período letivo e o de férias escolares do Instituto Federal Goiano - Campus Urutaí, visto que grande parte da população do município de Urutaí é composta por estudantes do referido Instituto. Assim, confrontando os valores obtidos durante os diferentes períodos foram determinados os impactos ocasionados pela população flutuante na geração de resíduos, fornecendo subsídios para uma gestão integrada e eficiente.

A fim de fornecer subsídios para implementação de coletas seletivas, além da composição gravimétrica, determinou-se os prejuízos econômicos decorrentes da atual deposição final dos resíduos, levantando-se o valor de comercialização dos resíduos com potencial reciclável por meio de pesquisas de mercado, bem como a geração de emprego e renda, até adequação ambiental.

\section{RESULTADOS E DISCUSSÃO}

Segundo o IBGE (2014), a população fixa de Urutaí é de 3.070 habitantes, sendo 70,8\% residentes na zona urbana e, destes, $80 \%$ são atendidos pelo sistema de coleta de resíduos (1.739 pessoas). Todavia, em virtude do período escolar, a população tem um acréscimo de aproximadamente $13 \%$ (1.965 pessoas).

No Quadro 1 estão apresentados os resultados da composição gravimétrica dos resíduos sólidos gerados pela população urbana de Urutaí-GO, por classe de materiais recicláveis, obtidas ao longo do período experimental.

Segundo MONTEIRO et al. (2001), a composição gravimétrica brasileira média é composta por $3 \%$ de plástico, $4 \%$ de metal, $3 \%$ de vidro e $25 \%$ de papel. Assim, analisando o Quadro 1, verifica-se que apenas os valores de metal, para o período letivo e, metal e vidro, no período de férias, estão próximos daqueles valores considerados como médios das cidades brasileiras.

Fica evidente a importância do levantamento gravimétrico "in loco", visto que em relação aos valores médios da literatura, foram determinados valores $248,97 \% ; 7,25 \% ; 3,25 \%$ e $-47,32 \%$, para o período letivo e, $126,87 \% ;-52,11 \% ;-37,42 \%$ e, $-66,66 \%$, para o período de férias, para os componentes, plástico, metal, vidro e papel, respectivamente. Dessa forma, ao considerarmos

Quadro 1. Composições gravimétrica (\%) dos resíduos sólidos urbano de Urutaí-GO, obtidos em $1 \mathrm{~m}^{3}$ de amostra

\begin{tabular}{c|ccc|c|ccc|c}
\hline \multirow{2}{*}{ Material } & \multicolumn{9}{|c}{ Período letivo } \\
\cline { 2 - 9 } & $07 /$ mai & $16 /$ mai & $15 /$ jun & Média & $16 /$ jul & $27 /$ jul & $08 /$ ago & Média \\
\hline Plástico & 10,71 & 8,57 & 12,12 & 10,47 & 6,20 & 7,26 & 6,95 & 6,81 \\
Metais & 4,29 & 2,86 & 6,06 & 4,29 & 2,33 & 1,28 & 2,14 & 1,92 \\
Vidros & 2,86 & 4,90 & 4,55 & 4,13 & 0,78 & 2,99 & 3,74 & 2,50 \\
Papel & 14,29 & 9,80 & 16,16 & 13,17 & 7,36 & 8,55 & 9,09 & 8,33 \\
Descarte & 67,86 & 73,88 & 61,11 & 68,07 & 83,33 & 79,91 & 78,07 & 80,44 \\
\hline
\end{tabular}


os valores de literatura para o dimensionamento de aterro e sua estimativa de vida útil, bem como para a implantação de um sistema de coleta seletiva, poderíamos incorrer em erros de superestimava ou subestimativa.

De acordo com GOMES et al. (2007), os valores da composição gravimétrica encontrada em Ouro Preto -MG foram de 2,15\% de metais, $10,60 \%$ de plásticos, $20,72 \%$ de papeis. Logo verifica-se que o quantitativo de plástico, obtido durante o período letivo, e o de metal, obtido no período de férias, estão bastante próximos aos encontrados no município de Ouro Preto-MG, sendo os demais materiais apresentaram valores bastante diferenciados, evidenciando que mesmo se tratando de duas cidades com população flutuante, a composição gravimétrica é especifica para cada tipo de população, sendo necessária a determinação de tais quantitativos para cada localidade.

Também, de acordo com o Quadro 1, verifica-se o efeito da sazonalidade provocado pela ausência dos estudantes, determinando redução de 34,99\%; $55,35 \% ; 39,39 \% ; 36,72 \%$ para os componentes, plástico, metal, vidro e papel, respectivamente e, acréscimo de $18,17 \%$, para o material descarte. Dessa forma, torna-se imprescindível, para um bom planejamento da estrutura disponível ao sistema de coleta de resíduos do município, o conhecimento destes dados para que não ocorra sobrecarga num período e ociosidade em outro.

Verificou-se que o município adota sistema de coleta de resíduos em dias alternados, qual seja, segundas, quartas e sextas feiras, com a inexistência da separação dos resíduos. Segundo RIBEIRO \& BESEN et al. (2007), a separação dos materiais recicláveis cumpre um papel estratégico na gestão integrada de resíduos sólidos sob vários aspectos: estimula o hábito da separação do lixo na fonte geradora para o seu aproveitamento, promove a educação ambiental voltada para a redução do consumo e do desperdício, gera trabalho e renda e melhora a qualidade da matéria orgânica para a compostagem. Assim, durante o período letivo e de férias foram feitas as seguintes observações quanto ao sistema de coleta:

Período de aula: duas viagens por dia de coleta, sendo cada viagem com duas carretas com capacidade volumétrica de três metros cúbicos cada. Assim durante o período letivo foram coletados, diariamente, 12 metros cúbicos de resíduos gerados.

Período de férias: duas viagens por dia de coleta, sendo a primeira com duas carretas e a segunda com uma carreta. Assim, durante o período de férias foram coletados, diariamente, 9 $\mathrm{m}^{3}$ de resíduos gerados.

Dessa forma, como o sistema de coleta é realizado três vezes por semana e, considerado os volumes coletados diariamente, verifica-se que, para o período das aulas, são produzidos, mensalmente $144 \mathrm{~m}^{3} \mathrm{e}$, para o período das férias, este volume reduz a $108 \mathrm{~m}^{3}$, o que representa uma redução de $25 \%$ no volume de resíduos. Pode-se, então, afirmar que a contribuição da população flutuante é bastante notável na geração de resíduos domiciliares, sendo, portanto, imprescindível considerar o impacto da presença ou ausências dos estudantes para um adequado planejamento do sistema de coleta dos resíduos sólidos urbano.

Uma vez conhecido o volume gerado nos diferentes períodos, foi determinada a quantidade de cada material a ser conduzida ao lixão municipal. No Quadro 2, estão apresentadas as quantidades esperadas para cada material no lixão municipal.

Quadro 2. Quantidade de resíduos gerados esperados, por classe, para os diferentes períodos avaliados

\begin{tabular}{c|ccc|ccc}
\hline \multirow{2}{*}{ Material } & \multicolumn{3}{|c|}{ Período letivo } & \multicolumn{3}{c}{ Período de férias } \\
\cline { 2 - 7 } & $\begin{array}{c}\text { Média em } \\
1 \mathrm{~m}^{3}(\mathrm{~kg})\end{array}$ & $\begin{array}{c}\text { Quantidade } \\
\text { diária }(\mathrm{kg})\end{array}$ & $\begin{array}{c}\text { Quantidade } \\
\text { mensal }(\mathrm{kg})\end{array}$ & $\begin{array}{c}\text { Média em } \\
1 \mathrm{~m}^{3}(\mathrm{~kg})\end{array}$ & $\begin{array}{c}\text { Quantidade } \\
\text { diária }(\mathrm{kg})\end{array}$ & $\begin{array}{c}\text { Quantidade } \\
\text { mensal }(\mathrm{kg})\end{array}$ \\
\hline Plástico & 22,50 & 270,00 & 3240,00 & 15,30 & 137,70 & 1652,40 \\
Metais & 9,30 & 111,60 & 1339,20 & 4,30 & 38,70 & 464,40 \\
Vidros & 9,00 & 108,00 & 1296,00 & 5,30 & 47,70 & 572,40 \\
Papel & 28,60 & 343,20 & 4118,40 & 18,60 & 167,40 & 2008,80 \\
Descarte & 148,00 & 1776,00 & 21312,00 & 182,60 & 1643,40 & 19720,80 \\
\hline Total & $\mathbf{2 1 7 , 6 0}$ & $\mathbf{2 6 0 8 , 8 0}$ & $\mathbf{3 1 3 0 5 , 6 0}$ & $\mathbf{2 2 6 , 3 0}$ & $\mathbf{2 0 3 4 , 9 0}$ & $\mathbf{2 4 4 1 8 , 8 0}$ \\
\hline
\end{tabular}


Com base no Quadro 2, espera-se, em relação ao período letivo, uma redução mensal na geração de resíduos de $22 \%$, o que corresponde a $1,8 \%$ diariamente. Estes resultados corroboram com aqueles apresentados anteriormente (composição gravimétrica) e, deixa evidente a importância do efeito da sazonalidade na geração de resíduo no município de Urutaí o que, até o presente momento, tem sido relegado a segundo plano.

Ainda de acordo com o Quadro 2, foram determinados o peso especifico aparente do resíduo gerado em Urutaí, sendo que durante período escolar foi obtido, em média, $217,60 \mathrm{~kg}$ de resíduo para cada $1 \mathrm{~m}^{3}$ e, 226,30 $\mathrm{kg}$ de resíduo para cada $1 \mathrm{~m}^{3}$ de resíduo, para o período de férias. Dessa forma, considerando-se o efeito da sazonalidade, houve um aumento de $4 \%$ no peso.

A partir da quantidade diária de resíduo gerado (Quadro 2) e da população existente, foram determinados a geração per capta. Assim, para o período letivo, foram gerados $0,75 \mathrm{~kg} \mathrm{hab}^{-1} \mathrm{dia}^{-1}$, enquanto para o período de férias, considerando uma população flutuante em torno de $13 \%$, esta geração passou para $0,66 \mathrm{~kg} \mathrm{hab}^{-1} \mathrm{dia}^{-1}$, o que representa uma redução de mais de $12 \%$.

Em relação a umidade, verificou-se que, em média, os resíduos conduzidos ao lixão municipal apresentam teor de umidade de $35,00 \% \pm 5 \%$. Já a compressividade média foi de $29,30 \% \pm 7,8 \%$.

A partir dos dados de volume de resíduos gerados para cada classe de recicláveis de interesse, estimaram-se os valores que cada reciclável poderiam gerar de retorno financeiro, caso fossem comercializados no mercado. Nos Quadros 3 e 4, estão apresentados os valores unitários e totais para os diferentes materiais recicláveis, para os diferentes períodos avaliados.

Conforme tabelas de preços dos compradores credenciados na ASCICLO (Associação das Empresas de Reciclagem do Estado de Goiás) verificou-se que, mensalmente, são desperdiçados R\$ 2.431,44 no período letivo (ou 3,4 salários mínimos) e R $\$ 1.171,80$ (ou 1,6 salários mínimos) no período de férias escolares. Dessa forma, considerando o papel social das cooperativas de reciclagem, bem como o aumento da vida útil do lixão, até adequação ambiental, bem como a limpeza urbana, o investimento em cooperativas de reciclagem ou de catadores torna-se indispensável e, quase, autossustentável.

Dentre todas as características encontradas durante a pesquisa, não podemos deixar de comentar sobre os resíduos descartados inadequadamente no lixão a céu aberto ou o que deveria ser um aterro

Quadro 3. Valor mensal dos resíduos- período letivo

\begin{tabular}{cccr}
\hline Material & Preço por $\mathrm{kg}$ & Peso $(\mathrm{kg})$ produzido por mês & \multicolumn{1}{c}{ Total por mês } \\
\hline Plástico & $\mathrm{R} \$ 0,50$ & 3240,00 & $\mathrm{R} \$ 1620,00$ \\
Metais & $\mathrm{R} \$ 0,25$ & 1339,20 & $\mathrm{R} \$ 334,80$ \\
Vidros & $\mathrm{R} \$ 0,05$ & 1296,00 & $\mathrm{R} \$ 64,80$ \\
Papel & $\mathrm{R} \$ 0,10$ & 4118,40 & $\mathrm{R} \$ 411,84$ \\
\hline & & Total & $\mathbf{R} \$ \mathbf{2 4 3 1 , 4 4}$
\end{tabular}

Quadro 4. Valor mensal dos resíduos - período de férias

\begin{tabular}{cccr}
\hline Material & Preço por Kg & Peso $(\mathrm{kg})$ produzido por mês & \multicolumn{1}{c}{ Total por mês } \\
\hline Plástico & $\mathrm{R} \$ 0,50$ & 1652,40 & $\mathrm{R} \$ 826,20$ \\
Metais & $\mathrm{R} \$ 0,25$ & 464,40 & $\mathrm{R} \$ 116,10$ \\
Vidros & $\mathrm{R} \$ 0,05$ & 572,40 & $\mathrm{R} \$ 28,620$ \\
Papel & $\mathrm{R} \$ 0,10$ & 2008,80 & $\mathrm{R} \$ 200,88$ \\
\hline & & Total & $\mathbf{R} \$ \mathbf{1 1 7 1 , 8 0}$
\end{tabular}


controlado. Foram encontrados diversos materiais como lixo eletrônico, restos de animais advindos de açougues, e até mesmo vacinas para animais e seringas, o que mostra a realidade atual.

\section{CONCLUSÕES}

Por meio das questões levantadas durante a pesquisa, foi possível verificar a situação atual em que se encontra o sistema de coleta de resíduos sólidos de Urutaí. Os resultados apontam:

- A necessidade de uma gestão integrada de resíduos, a fim de melhorar a qualidade dos recicláveis e otimizar a vida útil do terreno onde é feito o descarte dos resíduos (lixão), até regularização ambiental.

- O efeito da sazonalidade, que fica evidente nos diferentes períodos do ano conforme demonstrados neste estudo. Os dados levantados poderão servir para implantação de projetos para a atual e futuras gestões municipais, caso estes queiram implementar políticas adequadas e ambientalmente sustentáveis para a gestão integrada de resíduos sólidos.

- A indispensabilidade de um sistema de coleta seletiva, uma vez que o potencial de reciclagem poderá ser otimizado, aumentando o valor adquirido com a venda dos recicláveis. Todavia, para atingir este objetivo é necessária a participação de toda comunidade, uma vez que não se faz coleta seletiva somente de catadores e, sim, por meio da colaboração de todos.

- A adequação dos problemas relacionados ao acondicionamento de resíduos não poderá ser feita em curto prazo, já que é necessário a elaboração de um plano de manejo bem estruturado e, de um grande investimento, porém, os ganhos relacionados ao meio ambiente e a qualidade de vida dos moradores, torna qualquer projeto viável, uma vez que este já provou ser necessário.

\section{REFERÊNCIAS BIBLIOGRÁFICAS}

ASCICLO - Associação das Empresas de Reciclagem do Estado de Goiás. Disponível em: $<$ www.asciclo.org.br>. Acesso em 15 fev. 2015.
Cempre (Compromisso Empresarial para a Reciclagem). Coleta seletiva ainda é um desafio para o país. Cempre Informa, n.147, 2016. Disponível em: <http://cempre.org.br/cempreinforma/id/70/coleta-seletiva-ainda-e-um-desafiopara-o-pais--aponta-ciclosoft-2016>. Acesso em: 15 fev. 2015.

GOMES, F.P.A; MAIA, B.J.; REZENDE, A.; BARBOSA, S.L. Plano de gerenciamento integrado de resíduos sólidos urbanos. Ouro Preto, 2007. 267p.

INSTITUTO BRASILEIRO DE GEOGRAFIA E ESTATÍSTICA - IBGE. IBGE cidades 2014. Disponível em: < http://cidades.ibge.gov.br/xtras/ perfil.php?codmun $=522180>$ Acesso em: 15 fev. 2015.

LUSSARI, W.R.; LEAL, A.C.; TAKENAKA, E.M.M. A influência do problema de precarização no trabalho na relação entre o grupo de apoiadores e o modelo de gestão de uma cooperativa de reciclagem de resíduos sólidos. Periódico Eletrônico Fórum Ambiental da Alta Paulista, v.9, n.4, 2013.

MONTEIRO, J.H.P.; FIGUEIREDO C.E.M.; MAGALHÃES, A.F.; MELO, M.A.F.; BRITO, J.C.X.; ALMEIDA, T.P.F.; MANSUR, G.L. Manual de Gerenciamento Integrado de resíduos sólidos: Rio de Janeiro: IBAM, 2001, 200p.

OLIVEIRA, J.P.M.; OLIVEIRA, J.M.; BARRETO, E.S.; SILVA, S.S.; DA SILVA, S.S.; MARACAJÁ, P.B. Saúde/doença: as consequências da falta de saneamento básico. Informativo Técnico do Semiárido, v.9, n.2, p.23-29, 2015.

RIBEIRO, H.; BESEN, G.R. Panorama da coleta seletiva no Brasil: desafios e perspectivas a partir de três estudos de caso. Revista Interfacehs, v.2, n.4, p.1-18, 2007.

SCACABAROSSI, H.; CRUZ, C.F.; DA SILVA, G.D.F.N.; MUSSATO, O.B. Análise dos impactos ambientais causados pelo sistema de tratamento de resíduos líquidos na área de expansão urbana da cidade de Boa Vista-RR. Revista Geonorte, v.3, n.4, p.422-431, 2012. 